\title{
Age differential mortality in Spain, 1900-1991
}

\author{
Javier Llorca, M Dolores Prieto, C Fariñas Alvarez, Miguel Delgado-Rodriguez
}

\begin{abstract}
Study objective-To analyse the interindividual inequalities in mortality in Spain through the 20th century using the Gini coefficient, widely used as an income concentration index.

Design-Age mortality data were obtained from official publications of vital statistics and age and sex compositions were obtained from population census. The Gini coefficient was estimated. It can take values between 0 and 1 . Zero represents the situation in which all subjects die at the same age, whereas when all but one subject dies at 25 the index reaches a figure of 1 .

Main results-In both men and women there was a trend to decrease age differential mortality (from 0.26 to 0.16 for men and from 0.26 to 0.12 for women). Nevertheless, transitory increases were produced in 1918 (influenza epidemic), and in the period of the Civil War of Spain, showing a more important increase in the mortality of young people than that of the elderly. A new increase was observed through the second half of eighties; it resulted from an AIDS epidemic and motor vehicle injuries.

Conclusion-Inequalities in mortality in Spain have decreased through the 20th century.
\end{abstract}

(F Epidemiol Community Health 1998;52:259-261)

Premature mortality is one of the major consequences of inequality in health, independently of its origin (inequality in health services accessibility, life style, socioeconomic class, etc). Age adjusted death rates and life expectancy are not good measures to assess age differential mortality because both of them can increase if the whole mortality curve moves towards the right, although differential mortality remains constant.

Our aim is to use the Gini coefficient as a

Division of Preventive Medicine and Public Health, University of Cantabria, School of Medicine, Santander, Spain

Correspondence to: Dr J Llorca, Division of Preventive Medicine and Public Health, University of Cantabria School of Medicine, Av Cardenal Herrera Oria s/n,

39011-Santander, Spain.

Accepted for publication 5 June 1997 the evolution of age differential mortality in a country through time.

In this paper, we analyse the mortality differences according to age in Spain through the 20th century using the Gini index.

\section{Methods}

Age mortality data were obtained from Spain's National Institute for Statistics (SNIS), ${ }^{4}$ for each year from 1900 to 1991. Population data were obtained from decennial (1900, 1910, 1920, 1930, 1940, 1950, 1960, 1970, 1981, 1991) census population figures. ${ }^{5}$ Death rates per 100000 person years were calculated using intercensal populations estimated by exponential interpolation.

To calculate the Gini coefficient we need to know the age specific death rates for each year. They are obtained using the Gompertz function, ${ }^{6} R_{x}=R_{0} e^{a x}$, where $R_{x}$ is the specific mortality rate at age $x$. Several authors have proved the current mortality data fit the Gompertz function and they have discussed its consequences. $^{7-9}$ In a previous paper we have proved the Gompertz function works well in Spain for age 25 and over, ${ }^{10}$ therefore, we use only the mortality data for 25 years and over in this paper. The number of expected deaths at each age (until 100 years) is obtained applying the Gompertz function for each year to a cohort of 100000 people aged 25 years. Figure 1 shows a graph of the errors of the model for 1991 in Spain.

Let $M_{i}$ be the number of deaths at age $i$, and $T_{i}$ the number of person years lived by those who die at age $i . M_{i}$ can be derived from Gompertz function as $R_{i} N_{i}$ (where $N_{i}$ is the number of persons aged $i$ years. In analogous form, $T_{i}=$ $R_{i} N_{i}(i-25)$. As the Gompertz function is a monotonous and increasing function, it verifies that if $i>j$ then $M_{i}>M_{j}$.

For each age, we calculate:

$$
\begin{gathered}
M^{x}=\frac{\sum_{i=25}^{x} M_{i}}{\sum_{i=25}^{100} M_{i}} \\
T^{x}=\frac{\sum_{i=25}^{x} T_{i}}{\sum_{i=25}^{100} T_{i}}
\end{gathered}
$$

where $M^{x}$ is the number of accumulated deaths from age 25 to age $x$ divided by all the deaths accumulated between age 25 and age 100; and $T^{x}$ is the quotient of the number of person years yielded by people dying between age 25 and $x$ years divided by the total number of person years. 


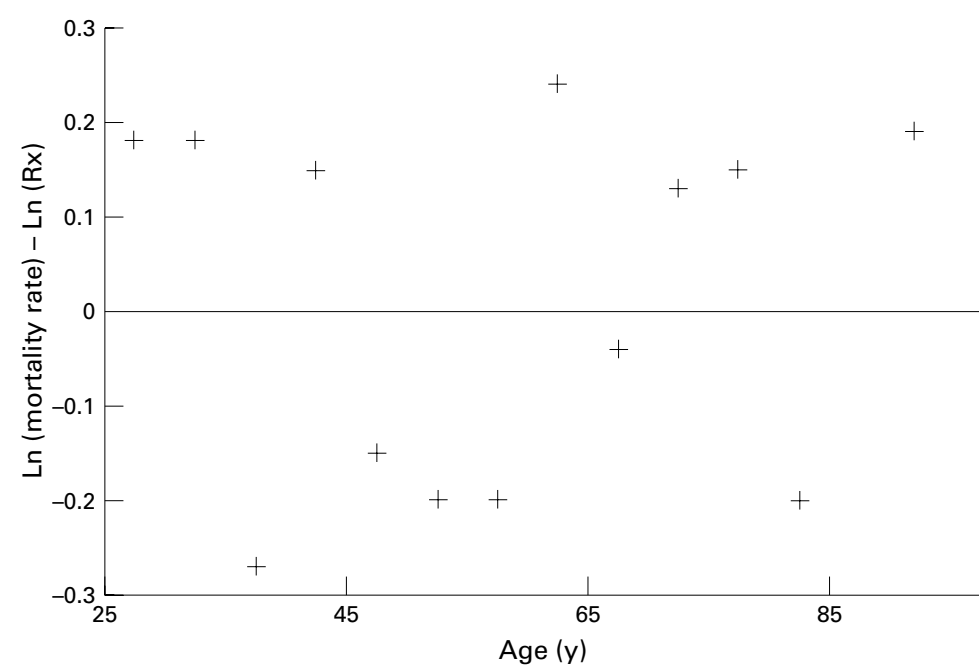

Figure 1 Errors of the Gompertz model for mortality data for the year 1991, Spain.

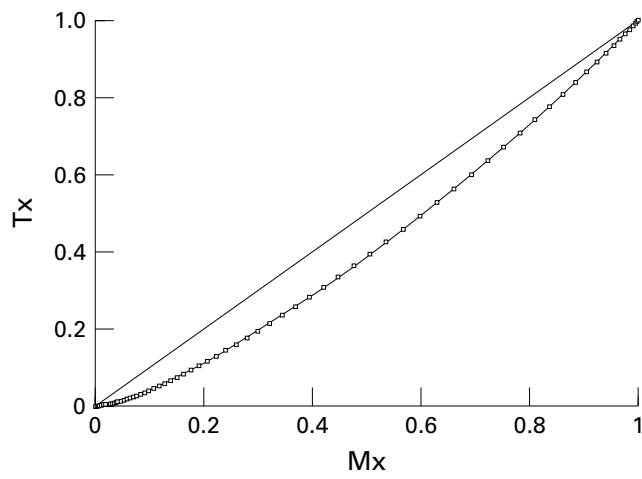

Figure 2 Construction of the Gini coefficient, Spain, 1980.

Both $M^{x}$ and $T^{x}$ range between 0 and 1 and they can be represented in a plot (fig 2 ) with $M^{x}$ in the $x$ axis and $T^{x}$ in the $y$ axis. A line through all the points can be drawn. This line is always below the diagonal line. The double of the area between the diagonal and the line obtained is called the Gini coefficient.

To estimate the Gini coefficient, the shape is integrated using the compound trapeze equa- tion (equation III); it is multiplied to two and substracted to 1 .

$$
\int^{99} T^{x} d M^{x}=\sum_{x=25}\left(M^{x+1}-M^{x}\right)\left(T^{x+1}-T^{x}\right) / 2
$$

If there are not inequalities in the mortality, all the points would be on the diagonal line, the graph would be a straight line and the Gini coefficient would be zero. If all the people except one person die at age 25 , the line would coincide with the $x$ axis and the Gini coefficient would be equal to one.

The Gini coefficient is a normalised adimensional index. It is robust because it is not affected by uniform increases/decreases in mortality of all age groups.

\section{Results}

Figure 3 displays the evolution of the Gini index in the Spanish men and women between 1900 and 1991. Throughout the period the differences among men have been greater than among women. In both men and women there is a trend to decrease age differential mortality (from 0.26 to 0.16 for men and from 0.26 to 0.12 for women). In the evolution of the index several segments can be observed: (a) from 1900 to 1917 the Gini coefficient decreased slowly (between 0.26 and 0.24 ) in both men and women; (b) in 1918 (year of the pandemic influenza), both sexes presented a great increase until 0.34; (c) from 1919 on the evolution is very different for men and women. In women the Gini coefficient decreased steadily until 1991, apart from a very slight increase during the Spanish Civil War (1936-1939) and a levelling off in the last four years of the period. In contrast, in men a slow decrease can be seen until 1935, a great increase between 1936 and 1941, a great decrease from 1942 to 1960, a very mild decrease from 1960 to 1987 , and a slight increase from 1988 on.

\section{Discussion}

Increase in life expectancy is believed to be a consequence of improvement in health status. However, life expectancy does not offer any

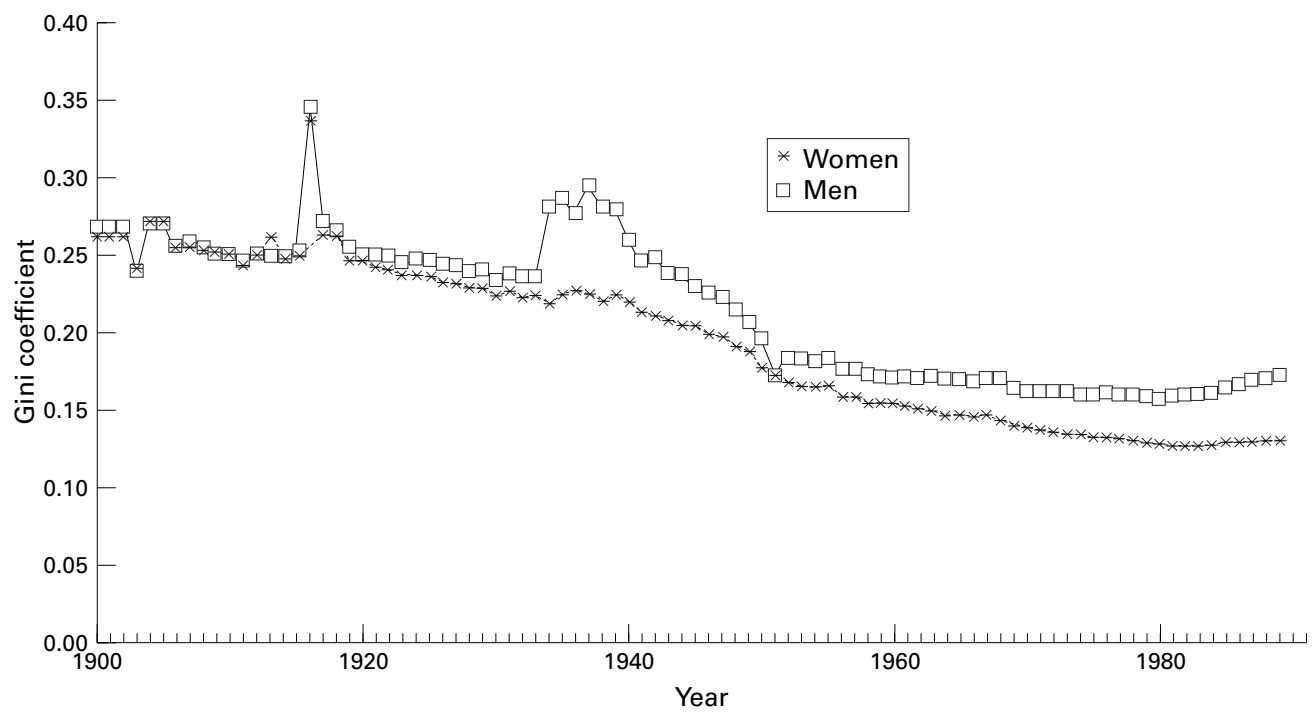

Figure 3 Gini coefficient, Spain, 1900-1991. 
information about whether this improvement is equal for different age groups. For example, the following can produce an increase in life expectancy: (a) a constant decrease in all age specific death rates; (b) a decrease in the death rates of the elderly only; and (c) a decrease in the death rates of young people only. In (a) interindividual differences remain constant, whereas in (b) the differences increase, and they decrease in (c) because more people live for longer; however, these three situations are recognised as a population improvement.

We have used the Gini coefficient to analyse interindividual inequality, but it cannot be used to analyse inequality resulting from specific factors influencing the mortality distribution. Nevertheless, some inferences can be made; for example, through this period an improvement in the access of least favoured socioeconomic groups to health services, education, and housing have occurred (for example, the proportion of the population who are literate has increased from $43.93 \%$ in 1900 to $96.75 \%$ in 1991 , population covered for the National Health Service has increased from $61 \%$ in 1960 to $94 \%$ in 1991, proportion of active population working in the building sector has increased from $3 \%$ in 1900 to $11 \%$ in 1991 , people living in rural areas has decreased from $60 \%$ in 1900 to $23 \%$ in 1991 , and people with university degrees have increased from 6000 per year aroung 1960 to 76399 in 1991). ${ }^{11}$ These facts can explain the decrease of Gini coefficient through this century.

The large increase for both sexes in 1918 could be because of the influenza epidemic. The increase of mortality resulting from influenza was proportionally greater in young people than in other adults. The same can be inferred for men during Spanish Civil War and post-war years. The evolution in men after 1960 is not as would be expected. Men should show a similar decrease to that of women as a general consequence of the improvement in health status. However, the Gini coefficient did not decrease for 20 years, showing that mortality in young men did not diminish in the same proportion as in women. Migration (about one million people, $75 \%$ men, and half a million 15-34 year old men) to other European countries (mainly Germany) occurred in Spain during the $1960 \mathrm{~s}^{12-14}$ This migration, which affected healthy young men of lower socioeconomic class most probably produced an artefact: these subject were excluded from the denominators and it prevented a decrease in the Gini index.

We have not explored any specific cause of death in the trend analysis of the Gini coefficient. A consideration of this point should explore those causes that change diferentially through time in young people than in the elderly. For example, mortality because of infectious diseases has decreased $7 \%$ per year in 25-29 year old men from 1951 to 1980 , whereas in 65-69 year old men this decrease
KEY POINTS

- The Gini index allows the analysis of age differental mortality through time.

- The age differential mortality has been reduced more in women than in men in Spain.

- The Gini index is not appropriate for the analysis of specific causes of death.

has been lower, 3\% per year for the same period. ${ }^{4}$ To estimate the Gini coefficient we need to acount for the whole mortality. The analysis of individual causes is difficult because if the subjects died from other causes apart from the one being studied is subjected to competing risks. For individual causes of death, the estimation of the Gini coefficient requires that the whole population would die of the same cause. Thus, the use of the Gini coefficient to study individual causes of death is not feasible.

A premature mortality increase during the 1980s, mainly because of an increase in AIDS and motor vehicle injuries, has been observed. ${ }^{4}$ We believe this is the reason for the increase in the Gini coefficient for the period 1987-1991 and not a deterioration in health services accessibility or a worsening in socioeconomic conditions.

In conclusion, inequalities in mortality in Spain have decreased through the 20th century. This decrease has been more important for women, showing a greater decrease in premature mortality in women than in men.

1 Gini C. Sulla misura della concentrazione e della variabilita dei caratteri. Atti del Reale Istituto Veneto di Scienze, lettere ed Arti, 1914;LXXII,parte II:1203-48.

2 Le Grand J. An international comparison of distributions of age-at-death. In: Fox J, ed. Health inequalities in European countries. Brookfield, USA: Gower, 1989:75-91.

3 Leclerc A, Lert F, Fabien C. Differential mortality: some comparisons between England and Wales, Finland and France, based on inequality measures. Int f Epidemiol France, based on

4 Movimiento Natural de la Población Española. Madrid: Instituto Nacional de Estadística, from 1900 to 1991

5 Censo de la Población Española. Madrid: Instituto Nacional de Estadística, 1900, 1910, 1920, 1930, 1940, 1950, 1960, 1970, 1981, 1991 .

6 Gompertz B. On the nature of the function expressive of the law of human mortality. Phil Trans R Soc Lond 1825;A115: 513-85.

7 Riggs JE. Longitudinal Gompertzian analysis of adult mortality in the US, 1900-1986. Mech Ageing Dev 1990;54: 235-47.

8 Shao Z, Gao W, Yao Y, Zhuo Y and Riggs JE. The dynamics of aging and mortality in the People's Republic of China, 1957-1990. Mech Ageing Dev 1993;67:239-46.

9 Juckett DA and Rosenberg B. Comparison of the Gompertz and Weibull functions as descriptors for human distributions and their intersections. Mech Ageing Dev 1993;69:131 .

10 Prieto MD, Llorca J, Delgado-Rodriguez M. Longitudinal Gompertzian and Weibull analyses of adult mortalty in Spain (Europe), 1900-1992. Mech Ageing Dev 1996;90:3551 .

11 Anuario Estadístico. Madrid: Instituto Nacional de Estadística, 1912:1965, 1991.

12 Migraciones. Quinquenio 1961-1965. Madrid: Instituto Nacional de Estadística, 1990

13 Migraciones. Quinquenio 1966-1970. Madrid: Instituto Nacional de Estadística, 1991.

14 Anuario Estadístico. Madrid: Instituto Nacional de Estadística, 1968. 